\title{
Effect of Cross-Sectional Geometry on the Energy Absorption Capability of Unidirectional Carbon Fiber Reinforced Composite Tubes.
}

\author{
Y. Yang*, Y. Nishikawa, A. Nakai, U. S. Ishiaku, H. Hamada \\ *Address of corresponding author \\ Advanced Fibro-Science \\ Kyoto Institute of Technology \\ Matsugasaki, Sakyo-ku, Kyoto 606-8585, Japan \\ Tel \& Fax: +81-75-724-7844 \\ Email: yyuqiu04@kit.ac.jp
}

\begin{abstract}
Owing to the high strength and stiffness to weight ratios, composite materials are being adopted as a new energy absorption member. As a structural part, square tubes have geometrical advantage because the flat region can be assembled easily with other components in car body during the manufacturing process. In this study, unidirectional carbon fiber reinforced composite tubes with circular and square cross section were fabricated through pultrusion process and tested by quasi-static axial compression. The energy absorption capability of the circular tube was found to be superior to that of the square tube in terms of specific energy absorption. The energy absorbing mechanisms of square tubes were investigated on the basis of precise longitudinal cross-sectional observation through the crush zone tested in step-wise compression. It was found that the fracture mechanisms at the flat wall and the corner were different during crushing. The corner regions seem to support the crush load more effectively. A novel experimental method was designed to determine the energy absorption at the corners and the flat walls respectively. Finally, the difference in specific crushing stress between the corner and the flat wall region is discussed in terms of the influence of geometry.
\end{abstract}

\section{INTRODUCTION}

Modem fiber reinforced plastics (FRPs) date from around the time of the Second World War. Their outstanding mechanical properties led to their gradual adoption in many industry fields. Around the late 1970s, researchers turned their attention to the energy absorption properties of these materials and found that FRPs have great potential as energy absorption structural components instead of the traditional metal components in particular in the transportation sector owing to their high strength or stiffness to weight ratios $/ 1-8 /$.

Several authors have attempted to summarize the crushing performances of fiber-reinforced composite tubes under axial compression, with perhaps Hull /9/ and Farley \& Jones /10/ providing the most comprehensive treatments. Both sets of authors reported that two behavioral extremes have been observed for fiber-reinforced composite tubes undergoing progressive crushing. One of the extremes has been termed splaying crushing mode, which is characterized by very long inter-laminar, intra-laminar and parallel-to-fiber cracks with little or no fracture of the axial laminar bundles. Fragmentation crushing mode, on the other hand is characterized by wedge-shaped laminate cross-section, with one or more short inter-laminar and longitudinal cracks forming partial lamina bundles but with many fragments 
caused by fiber and resin fractures. Fairfull \& Hull /11/ investigated the energy absorbed by the frictional processes associated with axial compression of FRP tubes. Both the frictional effects within the crush zone of the material, and those caused by the crushed eomposite sliding across the test machine's platens were considered. It was found that frictional processes can account for more than $50 \%$ of the total energy absorbed by progressive crushing, even when very smooth platens are employed. Hamada et al. /12-14/ claim to have obtained the highest specific energy absorption of any FRP composite through the use of a thermoplastic polyetheretherketone (PEEK) matrix with carbon fibers. A value of $225(\mathrm{~kJ} / \mathrm{kg})$ has been recorded, which is more than twice that obtained for carbon-epoxy composites. This is attributed to the high fracture toughness of the PEEK matrix inhibiting crack growth, followed by the very high number of 4 fiber fractures within the crush zone that lead to significant energy absorption. Comparison of various fiber architectures has also been a subject of many studies /15-22/. These researches indicated that FRP tubes absorb energy through multi-micro fractures, which lead to the complication of the energy absorbing mechanisms during crushing process.

A representative load-displacement characteristic of a FRP tube undergoing stable crushing fracture is illustrated in Fig. 1. The load-displacement curve can be divided into two distinct regions. In region I the load $P$ increases rapidly and reaches a maximum $\mathrm{P}_{\max }$ before dropping to the lowest value $\left(\mathrm{P}_{\text {lowes }}\right)$. Region II i.e. from displacement $S_{a}$ to $S_{b}$, the load fluctuates in a quasi-random fashion with serrations of very small amplitude, which is well known as progressive crushing.

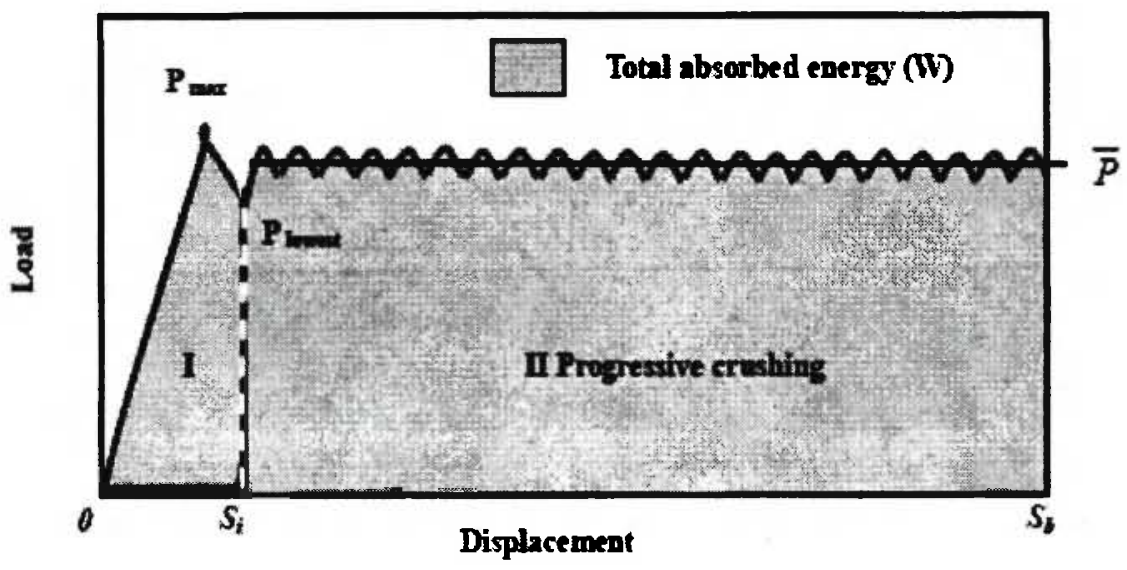

Fig. 1: Typical load displacement curve of a fiber-reinforced composite tube undergoing progressive crushing. I. Formation of crush zone; II. Progressive crushing.

The work done i.e. the total absorbed energy, $W$ is

$$
W=\int_{b}^{s_{b}} P d s
$$

Owing to the small displacement from 0 to $S_{i}$, the work done in the initial process can be ignored in the calculation. Therefore, the $\mathrm{W}$ will be

$$
W=\int_{b}^{S_{b}} P d s=\bar{P}\left(S_{b}-S_{i}\right)
$$


Where $\bar{P}$ is the average load during progressive crushing. The specific energy absorption, defined as the absorbed energy per unit mass of the crushed material, is

$$
\text { Specific energy absorption := } \frac{W}{A L \rho} \approx \frac{\bar{P}\left(S_{b}-S_{i}\right)}{A L \rho}=-\frac{\bar{P}}{A \rho}=\frac{\sigma}{\rho}
$$

Where, $A$ is the transverse cross sectional area of the wall of the tube, $L$ is the crush displacement, $\rho$ is the density of the material and $\sigma$ is the specific crushing stress. Specific energy absorption with a unit of $\mathrm{kJ} / \mathrm{kg}$ is one of the most important parameters that is often used to compare the energy absorption capabilities among various materials even though they may have different geometry, density, lay-up and so on.

Existing works on the energy absorption capability of FRPs have concentrated on the tubes with circular crosssection (circular tubes). However, recently, the tubes with square cross-section (square tubes) are found to be geometrically more practical as they can be assembled easily and steadily with other components during the manufacturing process of transport vehicles. Therefore, recently, the construction of database on the energy absorption of the square composite tube has been initiated /6, 23-25/. As regards the production of composite tubes, pultrusion is considered to be one of the most efficient manufacturing techniques; it facilitates the making textile preforms and impregnating them with resin to a final component in a continuous process to cut manufacturing cost /26-28/.

The aim of the current study is to understand the crushing performance of tubes with different geometries in the transverse cross section from the quasi-static compression test results of unidirectional carbon composite tubes with circular and square tubes. The difference of the fracture mechanism between the corner and the flat wall of the square tube is discussed based on the detailed observation of the longitudinal cross section through the crush zone. A novel experiment named as compression-segmentation-compression test was designed to investigate the difference in energy absorption between them. Finally, the geometrical moment of inertia was calculated as a typical parameter to represent the effect on the energy absorbing mechanisms in both parts.

\section{MATERIAL \& EXPERIMENTAL METHODS}

\section{Material}
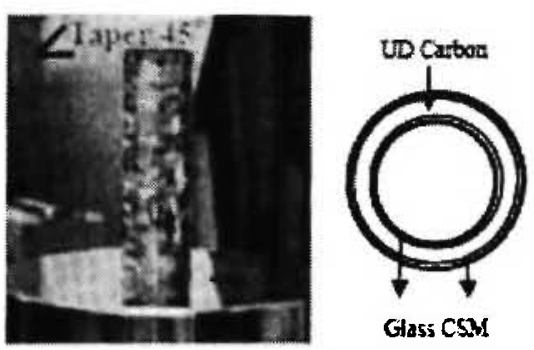

(a) Circular tube
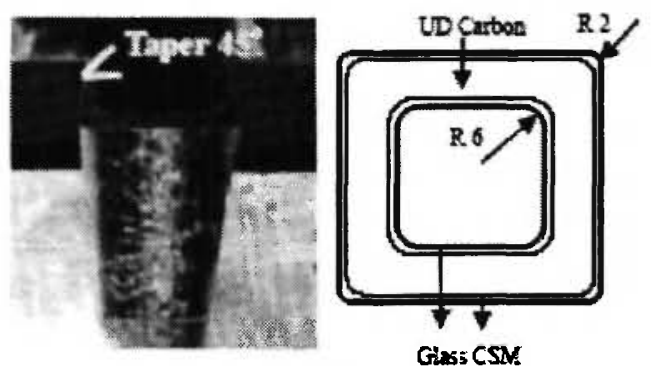

(b) Square tube

Fig. 2: Photographs of unidirectional carbon fiber/polyester FRP tubes fabricated by pultrusion process with different geometrical cross sections and schematic illustrations of the transversal cross sections illustrating the laminate, in particular, the inner and outer radii of the corner in a square tube. 
Two kinds of tubes with different geometrical cross sections, provided by Fukui Fibertech Co., Ltd, were fabricated by pultrusion method. Unidirectional carbon fiber bundles (Carbon UD) and unsaturated polyester resin were used as the reinforcement and matrix, respectively.

The photographs of the tubes and schematic illustrations of the transversal cross sections are shown in Fig.2. In order to obtain smooth surface and retain the shape during pultrusion fabrication, two layers of chopped strand glass mat (Glass CSM) were laid in the inner and outer parts of the 6 tube during pultrusion process. For the square tubes, as illustrated in Fig.2 (b), the inner and outer radii of the corners were 6 and $2 \mathrm{~mm}$, respectively.

The specifications of the tubes including reinforcement material, geometrical and physical parameters are given in Table 1. Both tubes were machined into individual specimens with a height of $100 \mathrm{~mm}$ and one end of each specimen was chamfered to a sharp edge with a taper angle of $45 \div$ in order to initiate progressive crushing $/ 1,29-30 /$.

Table 1

Specification of the unidirectional carbon/polyester FRP tubes with circular or square cross sections fabricated by pultrusion process.

\begin{tabular}{|c|c|c|c|c|c|c|c|}
\hline Type & UD carbon & $\begin{array}{c}\text { Glass CSM } \\
\left(\mathrm{gm}^{2}\right)\end{array}$ & $\begin{array}{c}\text { Outer Length } \\
(\mathrm{mm})\end{array}$ & $\begin{array}{c}\text { Thickness } \\
(\mathrm{mm})\end{array}$ & $\begin{array}{c}\text { Cross Section } \\
\left(\mathrm{mm}^{2}\right)\end{array}$ & $\mathrm{V}_{f}(\%)$ & $\begin{array}{c}\text { Density } \\
\left(\mathrm{g} \mathrm{cm}^{3}\right)\end{array}$ \\
\hline Circular & $\begin{array}{c}114 \text { bundles } \\
\text { of } 24 \mathrm{k} \mathrm{F}^{*}\end{array}$ & 300 & 30 & 3.0 & 255 & 50 & 1.47 \\
\hline Square & $\begin{array}{c}392 \text { bundles } \\
\text { of } 24 \mathrm{k} \mathrm{F}^{*}\end{array}$ & 450 & 50 & 4.2 & 797 & 51 & 1.56 \\
\hline
\end{tabular}

(24k F*: 24X1000 filaments)

\section{Compression experiment method}

Quasi-static compression test was performed on an INSTRON (4206) universal testing machine at a constant crosshead speed of $5.0 \mathrm{~mm} / \mathrm{min}$. The two kinds of tubes were axially crushed approximately $40 \mathrm{~mm}$ between parallel steel flat platens. Five repeat experiments were carried out for each kind of tube to verify the stability of energy absorption capability.

In particular, step-wise compression test was carried on square tubes. The test conditions were mostly the same as the former experiments but here specimens were crushed to the specified displacement. Step displacements were determined from the turning points in the load-displacement curve of the square tube. The first displacement was selected corresponding to the initiation load increasing stage. And the second and third displacements were decided corresponding approximately to the peak load and the lowest decrease point, respectively.

After that, the specimens crushed in step-wise compression tests and the one which was crushed to a displacement of $40 \mathrm{~mm}$, were cast in polyester resin to preserve the morphology of the crushed zone. Subsequently, some appropriate cross-sections were selected, polished and observed microscopically.

\section{Compression-segmentation-compression test}

Compression-segmentation-compression test was designed to determine the energy absorption at the corners and the flat walls regions of the square tube. The procedure of the test is 7 elaborated in Fig. 3. Firstly, quasi-static compression 
test was performed so that the crushed tube walls of the specimens were split into spreading fronds in order to realize progressive crushing. When the displacement reached about $30 \mathrm{~mm}$, the compression test was stopped and the compression plate was returned back. Secondly, the crushed specimen was brought out from the testing machine and segmented into parts by water-cooled diamond cutter according to the different cutting modes. Then the separated parts were compressed under the same condition as the initial stage, individually, as the third stage. The result of the compression-segmentation-compression tests is discussed based on the analysis of the load-displacement curves of the whole tube before cutting and those of its segmentations. Here, four cutting methods were employed. The aim of cutting modes 1 and 2 were to verify if the separated parts could continuous to realize progressive crushing. The total amount of loads of segmentations was almost of equal value as the average load during progressive crushing of the whole tube before cutting. On the other hand, the usage of cutting modes 3 and 4 was intended mainly to determine the energy absorption at the corners or flat wall regions, respectively. In order to simplify the calculation, the specific energy absorption of both regions was compared by using their specific crushing stresses instead due to the direct relationship shown in equation 3.

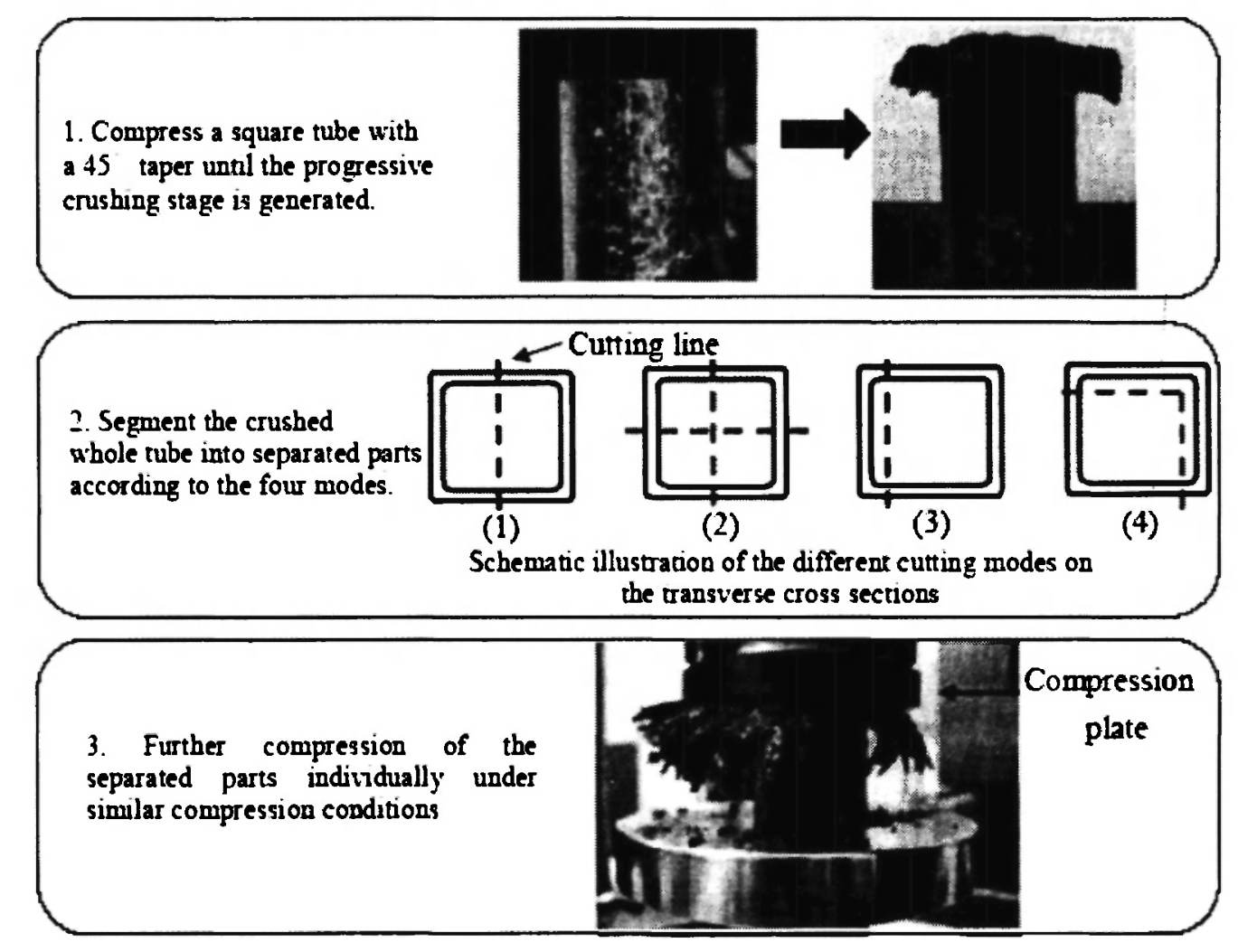

Fig. 3: Procedure of the compression-segmentation-compression test on the unidirectional carbon/polyester square tube.

\section{RESULTS AND DISCUSSION}

\section{Compression experiment of circular and square tubes}

With the advancement of compression steel platen, the taper part of the tube was initially bent to the inside of the tube and fractured. Then, the tube wall split into internal and external fronds, which looked like the spreading out of a 
flower. The photographs of the crushed specimens and the typical load-displacement curves are given in Fig. 4 and Fig. 5 , respectively. A common feature of both tubes is that the loads rapidly increased to a peak at the initial stage and then showed the characteristics of progressive crushing after $15 \mathrm{~mm}$ displacement. However, for the 8 square tubes, the loads displayed a drastic decrease from $97 \mathrm{kN}$ to $61 \mathrm{kN}$ just after the peak followed by a slow and gradual saturation before progressive crushing, which did not occur in the circular tubes.

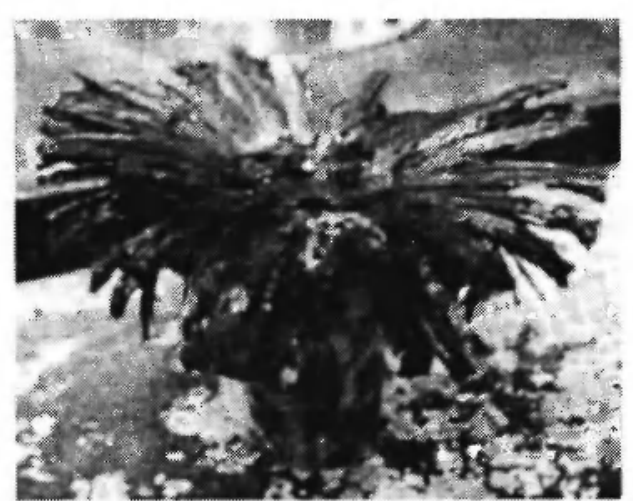

(a) Circular tube

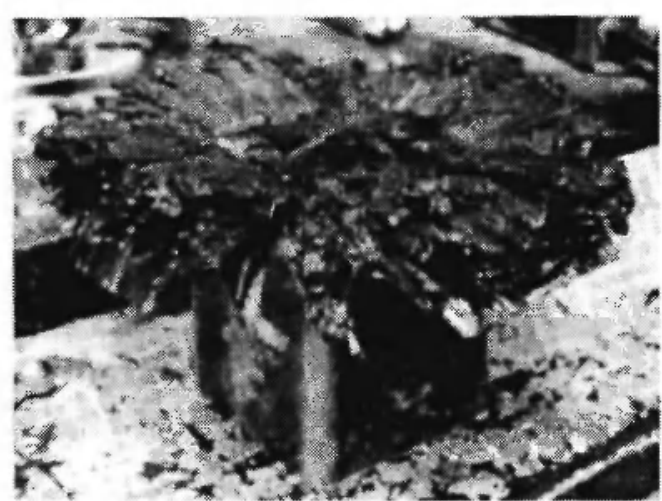

(b) Square tube

Fig. 4: Photographs of the unidirectional carbon/polyester FRP tubes crushed to a displacement of $40 \mathrm{~mm}$ at a speed of $5 \mathrm{~mm} / \mathrm{min}$, illustrated the spreading fronds.

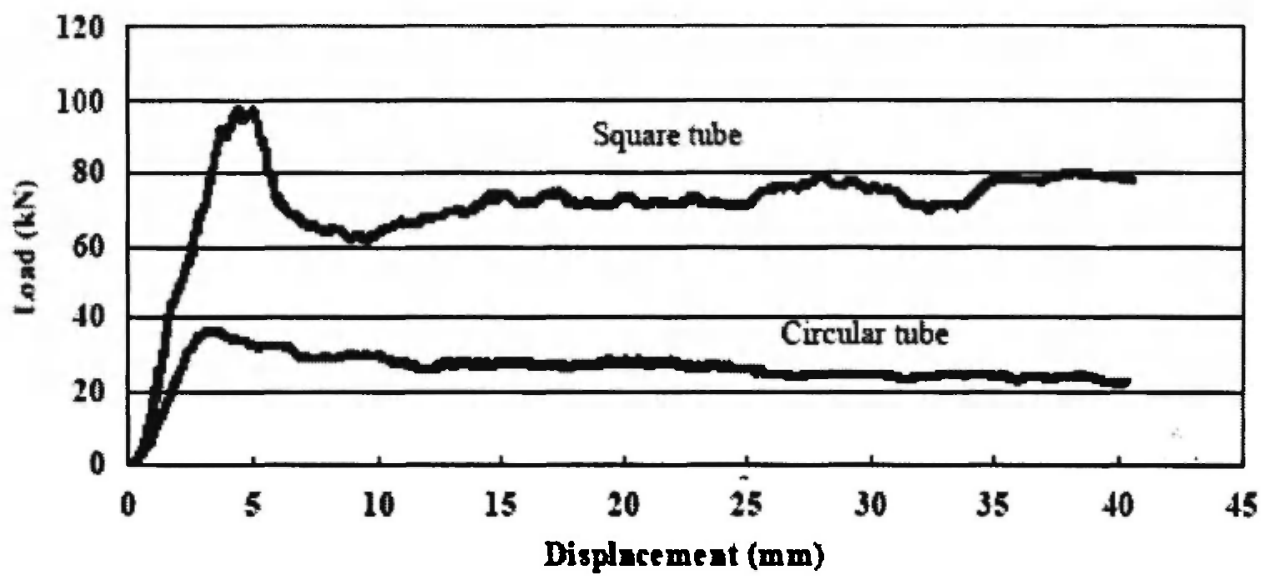

Fig. 5: Typical load-displacement curves for the unidirectional carbon/polyester circular and square tubes under quasistatic compression test at a speed of $5 \mathrm{~mm} / \mathrm{min}$.

The energy absorption capabilities of circular and square tubes were compared in terms of specific energy absorption calculated from the respective curves, which was $74.2 \pm 5.1(\mathrm{~kJ} / \mathrm{kg})$ for the circular tube and $55.3 \pm 4.6$ $(\mathrm{kJ} / \mathrm{kg})$ for the square tube. It could therefore be inferred that circular tubes have higher energy absorption capability than square tubes.

In the step-wise compression experiment on square tubes, the specific displacements were selected i.e. $3.5 \mathrm{~mm}$ as the first step, $5 \mathrm{~mm}$ and $10 \mathrm{~mm}$ as the second and third steps respectively corresponding to the initial load increasing stage, the peak and the lowest value of load. In order to clarify the energy absorbing mechanisms of square tube during crushing process, the cross section through crush zone were selected and observed. Here, considering the different 
geometrical regions in the transversal cross section of the square tube, longitudinal cross-sections in the middle of the flat wall and the corner were cut and assessed individually as shown in Fig. 6.

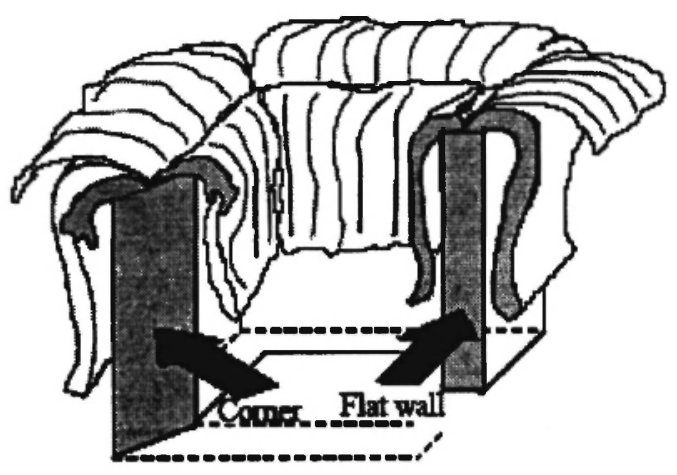

Fig. 6: Illustration of the selected axial cross sections, i.e. the middle of corner and flat wall region for observation.

The photographs and schematic diagrams of observation results are illustrated in Figs. 7-10. At the initial rapid load increase stage, the chamfered part was bent inwards, which caused shearing failure (Fig. 7). Compressive stresses paralleled to the fiber bundles induced longitudinal cracks, which were about $7.6 \mathrm{~mm}$ long at the corner and about $11.2 \mathrm{~mm}$ long at the flat wall. When the load reached peak value, the central crack in the flat wall advanced by about $3 \mathrm{~mm}$ and reached a length of $14.2 \mathrm{~mm}$, whereas, at the corner region, the main crack remained almost the same (Fig. 8). During the third stage, load dropped drastically from the peak to the lowest value while the central crack in the flat wall seemed to stop growing. However, many long and big delaminations were generated simultaneously. On the other hand, at the same stage, at the corner region, the wedge of debris, consisting of crushed fibers and resin, and small splittings were formed on the top side of the tube (Fig. 9). As Fig. 10 shows, after the wedge of the debris was 9 formed, with the advance of the compression plate, the tube wall was split and pushed apart as internal and external fronds along the long crack. The fronds experienced inter-laminar shear stresses, which result in delamination and splitting of the fronds into separate beams. There are more multi-micro fractures observed such as breaking of fiber bundles, which is thought to be generated with the resin broken also during bending of the fronds by tensile, compression and shear stresses. During progressive crushing, the cracks at both corner and flat wall were almost kept the same, which was considered to contribute to the stable crushing mode. Unlike the flat wall, where the tube wall split into pieces of internal and external fronds, the corner region fronds were split into smaller bits. It is thought that those fronds were sheared into many smaller beams at the corner region.

Based on these observations, the total absorbed energy during crushing process $U_{T}$ is summarized in equation 4 .

$$
U_{T}=U_{l c}+U_{d e}+U_{s f}+U_{b e n d}+U_{f f}+\text { others }
$$

where $U_{l c}$ is the energy required for longitudinal central crack of the pipe wall, $U_{d c}$ is the energy for delamination, $U_{s f}$ is the energy absorbed by the splitting of tube wall into pieces of fronds, $U_{b e n d}$ is the energy absorbed by bending of fronds, $U_{f f}$ is the energy required for fiber fracture and others include energies such as that associated with frictional heating. 


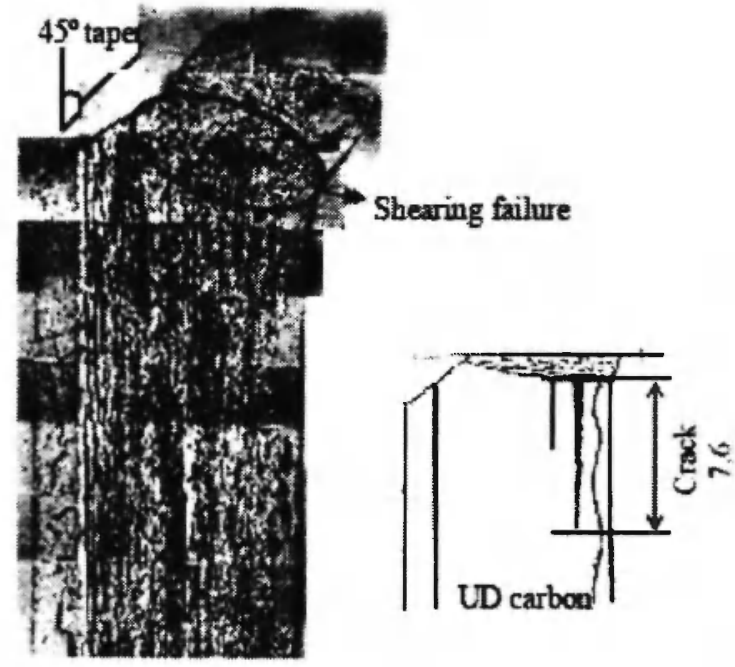

Outer

(a)

Inner

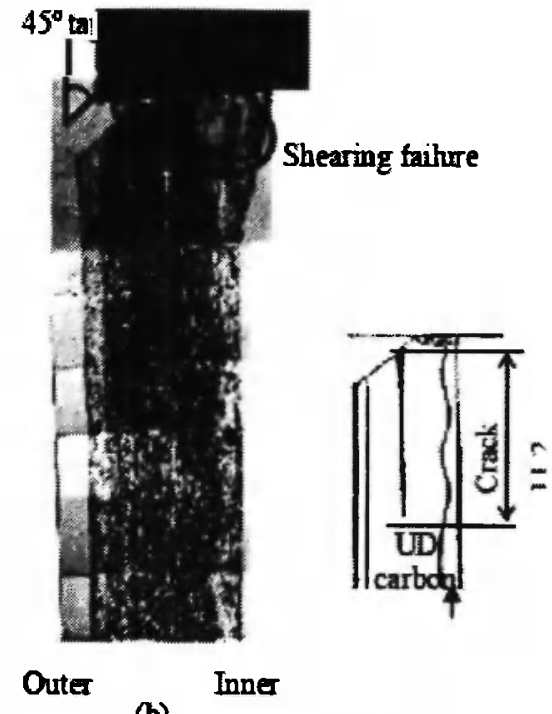

(b)

Fig. 7: Photographs and schematic illustrations of the axial cross sections of the corner and the flat wall region through the crush zone of the unidirectional carbon/polyester square tubes at the first stage of the step-wise experiment (displacement $=3.5 \mathrm{~mm})$. (a) Corner; (b) Flat wall.

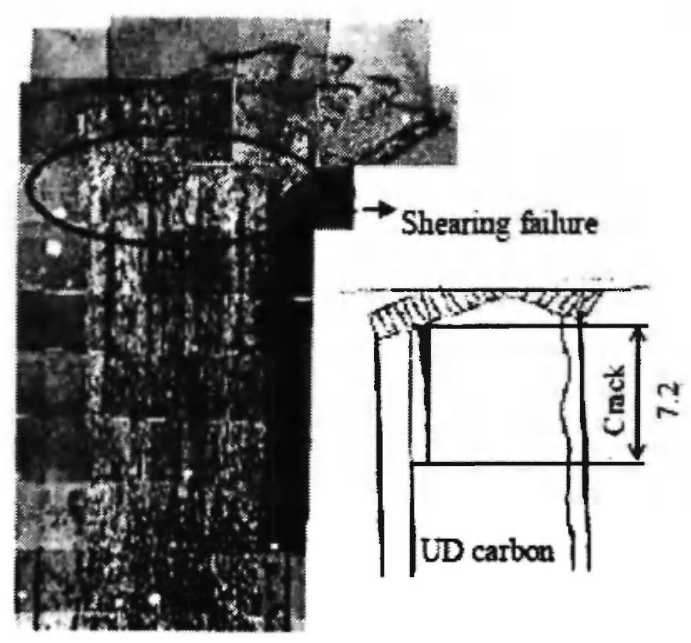

Outer

(a)

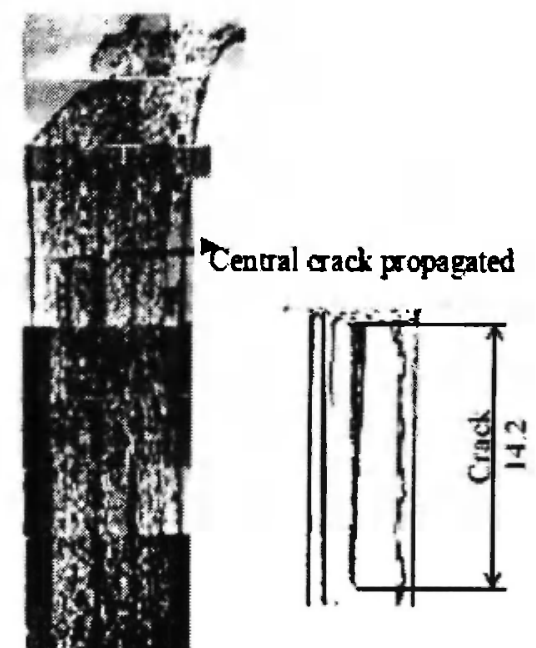

Outer

Fig. 8: Photographs and schematic illustrations of the axial cross sections of the corner and the flat wall region through the crush zone of the unidirectional carbon/polyester square tubes at the second stage of the step-wise experiment (displacement $=5 \mathrm{~mm}$ ). (a) Corner; (b) Flat wall.

The fracture mechanisms are created singularly or simultaneously even in an axial symmetry circular tube. Crushing behavior becomes more complicated in the square tube owning to the different geometries within the transversal cross section. The distinct fractures in the corner and flat wall region such as the different crack length during the initial crushing stages was considered as the main reason resulting in big load drop in the square tube before progressive crushing. With the square tube, the corner region seemed to support compression load more than the flat wall, because of the numerous smaller fractures involved. 


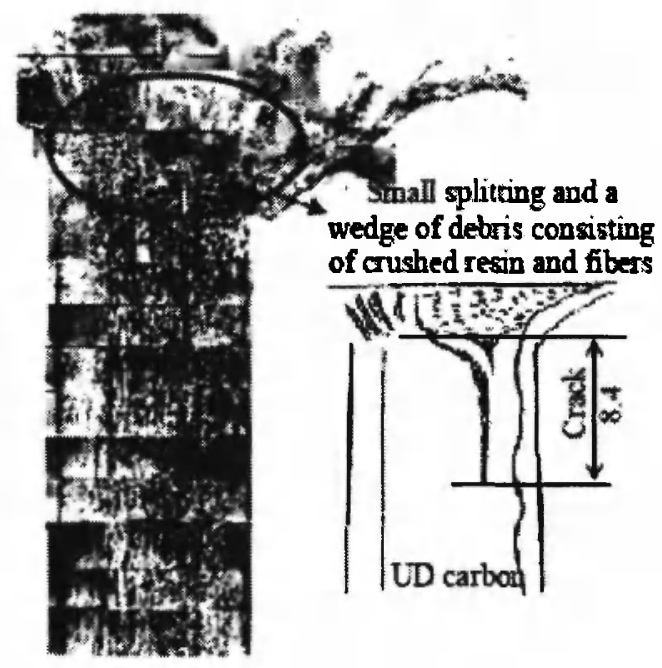

Outer

(a)

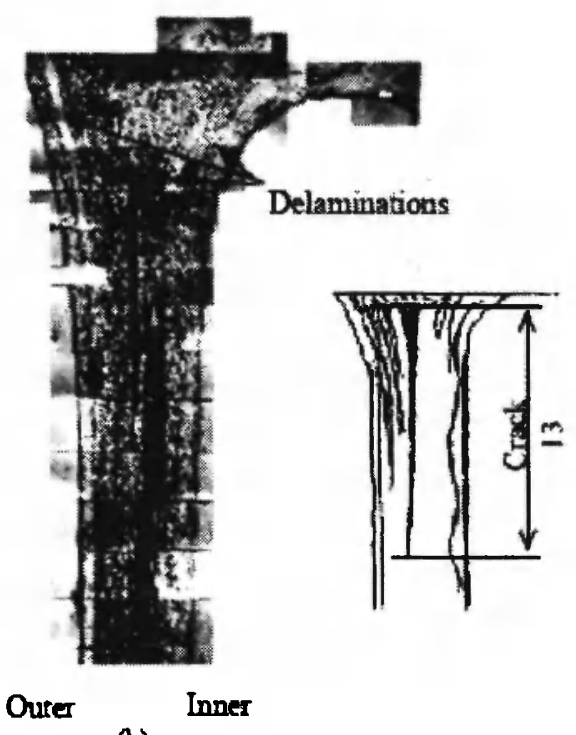

(b)

Fig. 9: Photographs and schematic illustrations of the axial cross sections of the corner and the flat wall region through the crush zone of the unidirectional carbon/polyester square tubes at the third stage of the step-wise experiment (displacement $=10 \mathrm{~mm})$. (a) Corner; (b) Flat wall.

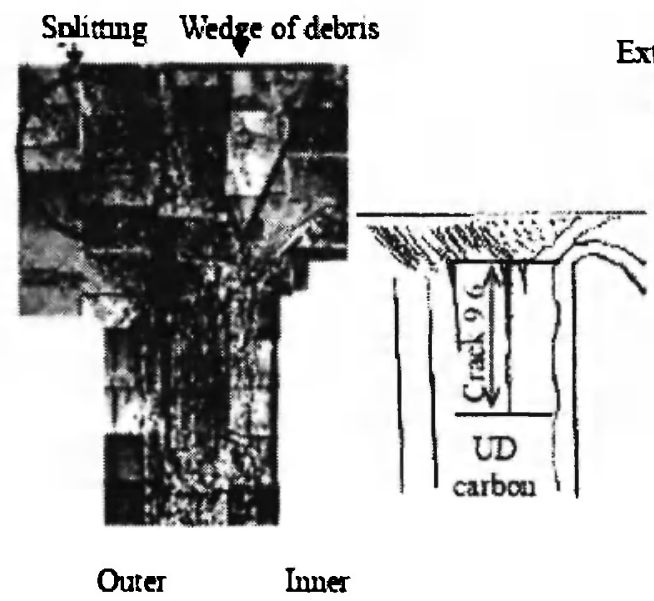

(a)

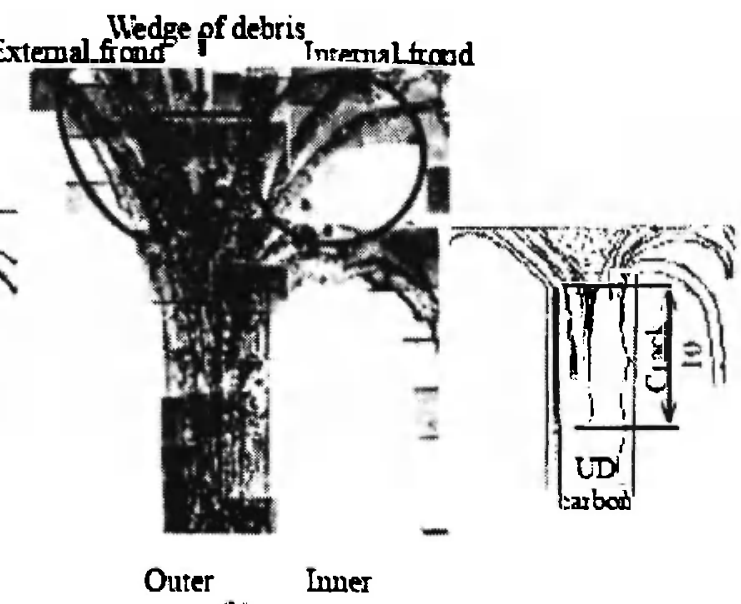

(b)

Fig. 10: Photographs and schematic illustrations of the axial cross sections of the corner and the flat wall region through the crush zone of the unidirectional carbon/polyester square tubes after compression test (displacement $=40 \mathrm{~mm}$ ). (a) Corner; (b) Flat wall.

\section{Compression-segmentation-compression test}

The results of cutting mode-1 illustrated in Fig. 3 (1) are elaborated in Fig. 11, including the schematic illustration of the cutting mode (a), the photographs of the tested segmentations (b) and the load-displacement curves (c). Like the compression experiments described above, at the initial stage, a crushing behavior similar to that of the whole tube occurred. That is, the load increased to a peak quickly via elastic deformation and then decreased before progressive crushing was generated. After nearly $30 \mathrm{~mm}$ displacement, the compression test was stopped and the whole tube was cut into two equal parts (Part A and B) along the cutting line shown in Fig. 11 (a). Both parts were compressed again under 
the same experimental conditions as the initial stage. The load-displacement curves of the two separated segmentations also showed the characteristics of progressive crushing during the later compression test. The load-displacement of $(A+B)$ was obtained by adding the loads of the both parts. The sum of loads $(P A+B)$ displayed clearly a trend similar to that $(\bar{P})$ of the whole tube before cutting, i.e.

$$
P_{a}+P_{b}=P_{A+B}=\bar{P}(k N)
$$

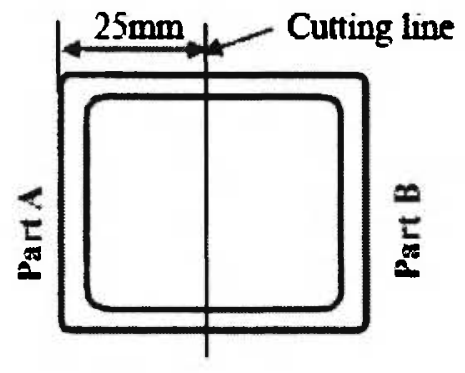

(a) Cutting mode-1
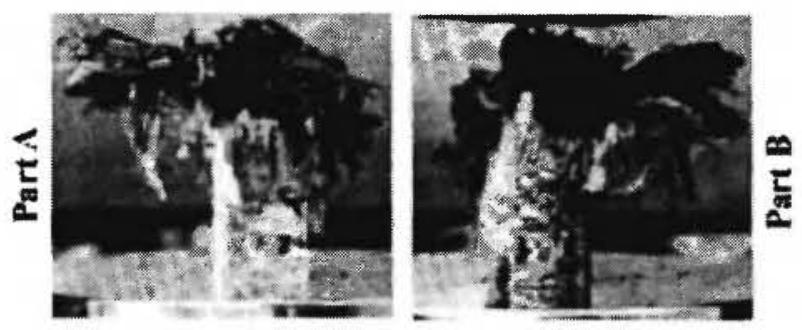

(b) Photographs of tested segmentations from side view

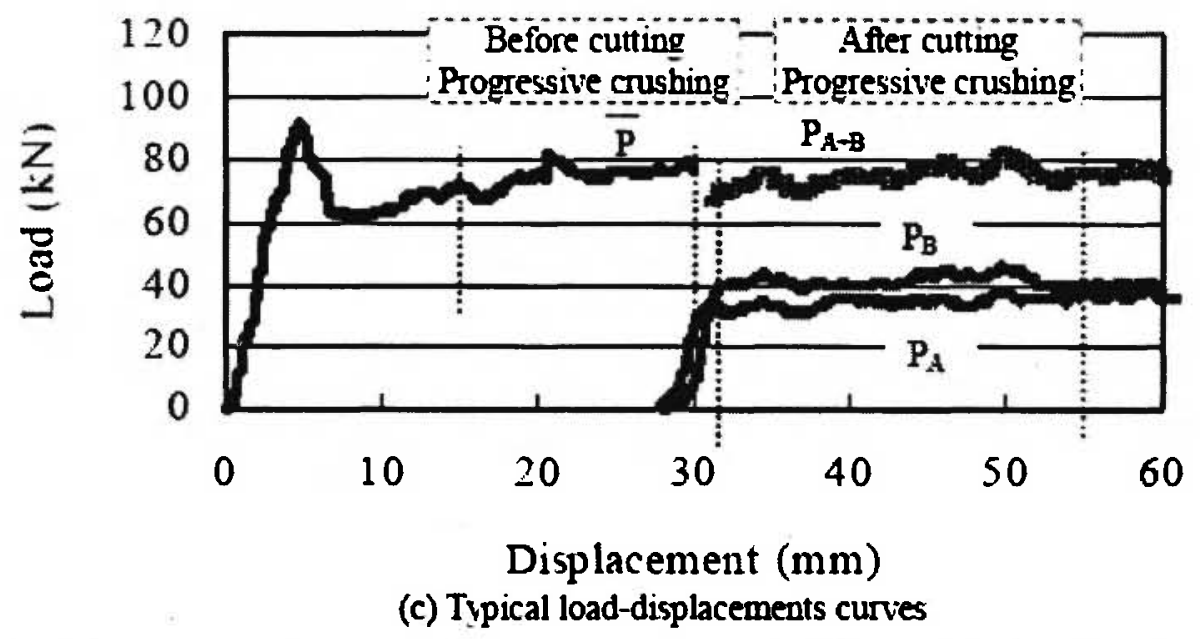

Fig. 11: Results of the cumpression-segmentation-compression test of the unidirectional carbon/polyester square tubes for cutting mode-1.

For a composite tube, the summation of the crushing performances of the segmentations did not change too much in comparison to the whole tube. The separated parts seem to be integral parts of the whole unit. The results of the compression-segmentation-compression test of cutting mode-2 illustrated in Fig. 3 (2) also yielded similar results. 


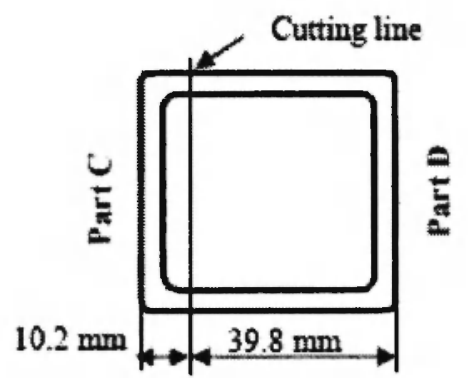

(a) Cutting method-3

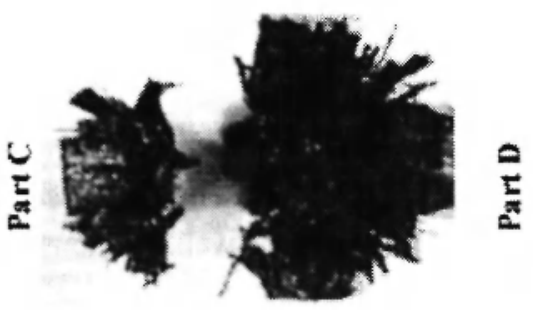

(b) Photographs of tested segmentations from top new

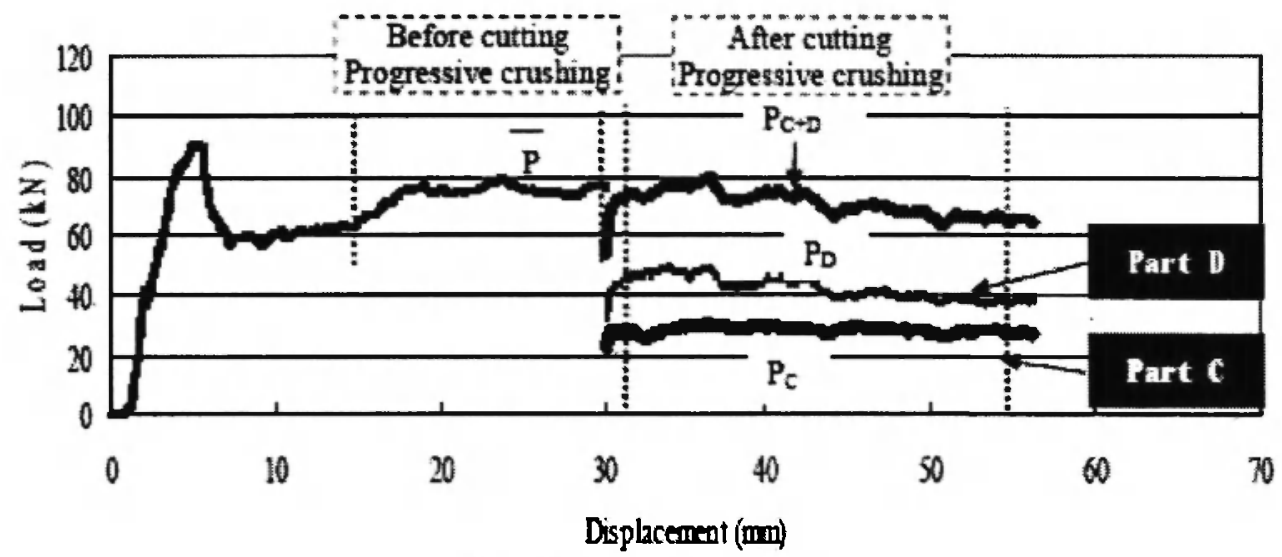

(c) Typical load-displacements curves

Fig. 12: Results of the compression-segmentation-compression test of unidirectional carbon/epoxy square tube for cutting mode-3.

The result of the test for cutting mode-3 illustrated in Fig. 3 (3) is presented in Fig. 12 to explain the calculation of the specific crushing stresses at the corners and the flat walls regions. The tube was segmented into Part C and Part D with a cutting line shown in Fig. 12 (a), which was determined according to the radius of $R_{\text {mner }}$ of a corner i.e. $6 \mathrm{~mm}$ and the thickness of the flat wall i.e. $4.2 \mathrm{~mm}$. Part $\mathrm{C}$ could be considered as two pieces of the corners plus one piece of the flat wall, while Part D consists of two pieces of the corners and three pieces of flat walls. The photographs of the segmentations at final crushing are shown in Fig. 12 (b). As shown in Fig. 12 (c), the averaged loads of part C and D during progressive crushing were named as $P_{c}$ and $P_{d}$, and the suppositional load of Part $C$ and Part $D, P_{c+d}$, was obtained by summing of these two parts, which was also found to have the similar value and trend with the mean load of the whole tube before cutting, i.e. $\bar{P}$. Then the parameters of the loads on both flat walls and corners regions are calculated as follows:

$$
\begin{aligned}
& P_{c}+P_{d}=P_{c+d}(k N) \\
& P_{d}-P_{c}=\frac{1}{2} P_{\text {flat wall }}(k N) \text { (half of the load on the flat walls region) } \\
& P_{c+d}-2\left(P_{d}-P_{c}\right)=\bar{F}_{c o r n e r}(k N) \text { (the load on the corners region) } \\
& \frac{P_{\text {corner }}}{S_{\text {corner }}}=\sigma_{\text {corner }} \text { (the specific crushing stress of the corners region }\left(\mathrm{kN} / \mathrm{m}^{2}\right)
\end{aligned}
$$


$\frac{P_{\text {flat wall }}}{S_{\text {flat wall }}}=\sigma_{\text {flat wall }}$ (the specific crushing stress of the flat walls region $(\mathrm{kN} / \mathrm{m} 2)$

Here, $P_{c}, P_{d}, P_{c+d}$ are the mean loads of segmented Part C, Part D, and a sum of these two parts, respectively. The compression loads on the flat walls and corners regions are named as $P_{\text {fat wall }}$ and $P_{\text {corner. }} S_{\text {cormer }}$ and $S_{\text {fat wall }}$ are the cross sectional areas of the corners and flat walls regions, respectively. Similarly, the results of the compressionsegmentation-compression test of the cutting mode-4 illustrated in Fig. 3 (4) were calculated and summarized in Table 2 together with that of cutting mode-3. It can be seen that the specific crushing stress of the corner region is higher than that of flat walls region by 1.7 times. That means, for a unit mass of the same material, the corners region can absorb $70 \%$ higher energy than the flat walls.

Table 2

Results of the compression-segmentation-compression tests on the unidirectional carbon/polyester square tubes for cutting modes 3 and 4 , illustrating that the specific crushing stress of the corners region is higher than that of the flat walls region by 1.7 times.

\begin{tabular}{|c|c|c|c|}
\hline & & $\begin{array}{l}\text { Cutting } \\
\text { modes } 3\end{array}$ & $\begin{array}{l}\text { Cutting } \\
\text { modes } 4\end{array}$ \\
\hline \multirow{3}{*}{ Comer } & P (load) & $37.88 \mathrm{kX}$ & $45.56 \mathrm{kN}$ \\
\hline & $S$ (Area) & $299.63 \mathrm{~mm}=$ & $301.06 \mathrm{~mm}^{2}$ \\
\hline & \multicolumn{2}{|c|}{ Specific crushing stress of the corners } & $\mathbf{m}^{2}$ \\
\hline \multirow{3}{*}{ Flat wall } & $P($ Load $)$ & $38.36 \mathrm{kN}$ & $39.83 \mathrm{kN}$ \\
\hline & $\mathrm{S}$ (Area) & $497.28 \mathrm{~mm}^{2}$ & $498.51 \mathrm{~mm}^{2}$ \\
\hline & \multicolumn{2}{|c|}{ Specific crushing stres of the flat walls } & \\
\hline
\end{tabular}

To understand the different specific crushing stresses, i.e. the energy management capacities between the corner and the flat wall regions in a square tube during crushing process, the energy absorbed by the bending of the fronds was considered as an important factor. On the other hand the flexural rigidity of a beam is often used to evaluate the behavior of the beam in bending. One of the major factors of flexural rigidity is the moments of inertia of the section areas of the beam, especially the principal moments of inertia in the case of similar materials, which reflect the affect of the sectional shape on the flexural rigidity. Therefore, the effect of geometries between the corner and the flat wall shown in Fig. 13 is discussed in terms of moments of inertia of the both regions with respect to the $\mathrm{Z}$ axis trough the centroid $\mathrm{C}$ of each cross section. The calculated results indicated that the moment of inertia of the corner was higher that that of the flat wall just by 1.7 times.

Flexural rigidity is considered to affect indirectly the propagation of the longitudinal crack and the bending of the fronds consequently. As a result of the increase of $U$ bend, the fronds would bend in a sharp radius and then high tensile or compressive stresses would be generated at the convex or concave side of the fronds, leading to more fiber fractures 
finally. The bending rigidity could be modified by the design of the radii of the corners. In other words, it is possible to improve the energy absorption capability of square tubes through the design of the corners specifically.

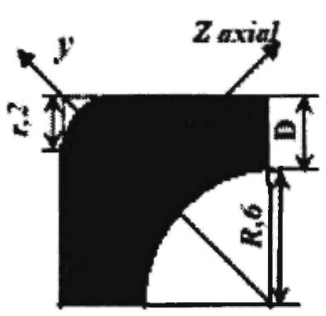

(a) Comer

$$
I_{Z \text { Corner }}=322.10 \mathrm{~mm}^{4}
$$

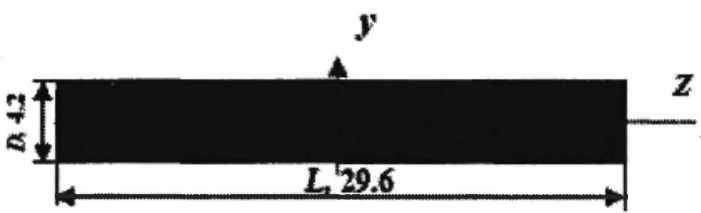

(b) Flat wall

$I_{Z \text { Flat wall }}=182.75 \mathrm{~mm}^{4}$

Fig. 13: Geometries of the corner and the flat wall in the unidirectional carbon/polyester square tube, illustrating that the moments of the inertia of corner are higher than that of the flat wall by 1.7 times.

\section{CONCLUSIONS}

In this study, the energy absorption performance of unidirectional carbon fiber reinforced composite tubes was investigated and the following conclusions were drawn:

1. The results indicate that square tubes had lower specific energy absorption than circular tubes.

2. Step-wise compression tests were employed on square tubes in order to determine the fracture mechanisms during the crushing process, and the resulting microstructures were examined microscopically. The results indicated that the multi-micro fractures between the corner and the flat wall region of a square tube were obviously different.

3. Compression-segmentation-compression test was designed to determine the energy absorption of the corners and the flat walls regions in a square tube, individually. Their energy absorption capability was compared in terms of specific crushing stresses. It was found that the stress of the corners region was higher as compared to that of the flat walls region. The difference in the specific energy absorptions (specific crushing stresses) was discussed in terms of flexural rigidity which reflects the affect of the geometrical shape.

\section{APPENDIX}

\section{Calculation of geometrical moments of inertia of the corner and flat wall region with respect to the $Z$ axes through the centroidal $C$}

In order to simplify the calculation, the corner region (Corner) was appropriately divided into several simple geometric areas according to the illustration below and the equation 10. 


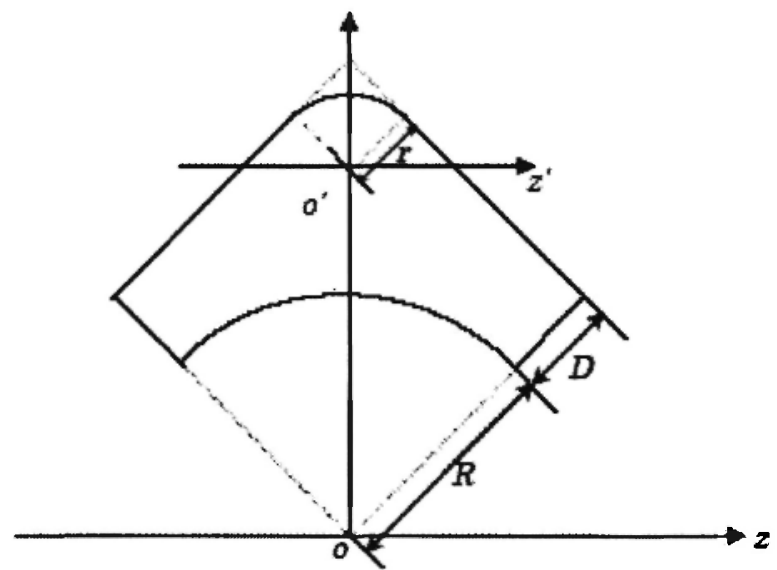

$$
\text { Corner }=S(R+D)-C(R)-S(r)+C(r)
$$

Here, $S(R+D), C(R), S(r), C(r)$ are assumed as a square with the side length of $R+D$ and the positive area, a square with the side length of $r$ and the negative area, one-fourth circle with the radius of $R$ and the negative area, and onefourth circle with the radius of $r$ and the positive area, respectively. Thus, the corner region can be considered as the summary of these four parts, and the moment of inertia of the corner region with respect to the $\mathrm{Z}$ axis through the centroid $\left(I_{z \text { Corner }}\right)$ is computed as equation $11 / 31-32 \%$.

$$
\begin{aligned}
I_{z \text { Corner }} & =\left\{\frac{(R+D)^{4}}{12}-\left[y_{c}-\frac{\sqrt{2}}{2}(R+D)\right]^{2}(R+D)^{2}\right\} \\
& -\left[\frac{\pi+2}{16} R^{4}-\left(y_{c}-\frac{4 \sqrt{2} \bar{R}}{3 \pi}\right)^{2} \frac{\pi R^{2}}{4}\right] \\
& -\left\{\frac{r^{4}}{12}-\left[y_{c}-\frac{\sqrt{2}}{2}(2 R+2 D-r)\right]^{2} r^{2}\right\} \\
& +\left\{\frac{\pi+2}{16} r^{4}-\left[y_{c}-\sqrt{2}(R+D-r)-\frac{4 \sqrt{2}}{3 \pi} r\right]^{2} \frac{\pi r^{2}}{4}\right\}
\end{aligned}
$$

Here, $R$, and $r$ are the inner and outer radii of the corner i.e. 6 and $2 \mathrm{~mm}$. $D$ is the thickness of the flat wall, i.e. $4.2 \mathrm{~mm}$.

On the other hand, the moment of inertia of the flat wall with respect to the $\mathrm{Z}$ axis through the centroid $\left(I_{Z \text { Flat wall }}\right)$ is calculated by equation $12 / 31 \%$.

$$
I_{Z \text { Flat wall }}-\frac{L D^{3}}{12}
$$

Here, $L$ is the length of the flat wall, i.e., $29.6 \mathrm{~mm}$.

\section{ACKNOWLEDGEMENT}

The authors thank Fukui Fibertech Co., Ltd for providing the fabricated specimens used in this study. 


\section{REFERENCES}

1. P.H. Thornton, Journal of Composite Materials, 13, (1979).

2. K.H. Lin and G. H. Mase, Journal of Engineering Materials and Technology, 112, (1990).

3. G.L. Farley, Journal of Composite Materials, 17, (1983).

4. V.P. McConnell, Automotive Composites: A Design and Manufacturing Guide, Ray Publishing, Wheat Ridge, $\mathrm{CO}$, 1, 6-7,1997.

5. S. Ashley, Mechanical Engineering, 118, 59, (1996).

6. C.M. Kindervater, Developments in the Science and Technology of Composite Materials, J. Fuller et al (Eds.), 1990.

7. R. Piellisch, SAMPE Journal, 32, 5, (1996).

8. H. Wallentowitz, and H. Adam, SAMPE Journal, 31, 5, (1995).

9. D. Hull, Composites Science \& Technology, 40, (1991).

10. G.L. Farley and R.M. Jones, Journal of Composite Materials, 26, (1992).

11. A.H. Fairfull and D. Hull, Structural Failure, T. Wierzbicki, and N. Jones (Eds.), 1988; p.255.

12. H. Hamada, J.C. Coppola, D. Hull, Z. Maekawa and H. Sato, Composites, 23, 4, (1992).

13. H. Hamada, S. Ramakrishna and H. Satoh, Composites, 26, 11 (1995).

14. H. Hamada, S. Ramakrishna and H. Sato, Journal of Composite Materials, 30, 8, (1996).

15. M.V. Karbhari, M., J. Falzon, and P. Herzberg, Journal of Composite Materials, 31, 12, (1997).

16. D. W. Schmueser and L. E. Wickliffe, Journal of Engineering Materials and Technology, 109, January (1987).

17. M.V. Karbhari, Journal of Composite Materials, 31, 10, (1997).

18. L.G. Farley, Journal of Composite Materials, 20, July (1986).

19. L.G. Farley, K.R. Bird and T.J. Modlin, Journal of the American Helicopter Society, April (1989).

20. H. Hamada, A. Nakai, K. Kameo, and N. Takeda, Proceedings of the Eighth Japan-U. S. Conference on Composite Materials, September 24-25, 1998.

21. J.A. Lavoie and J. Morton, NASA Contractor Report 4526, July (1993).

22. P.H. Thornton, and P.J. Edwards, Journal of Composite Materials, 16, November (1982).

23. N. Jonson, et al, ESD Advanced Composites Technologies, p.403-419, 1993.

24. P.H.Thornton and P.J. Edwards, Journal of Composite Materials, 16, (1982).

25. A.G. Mamalis, Y.B. Yuan and G.L. Viegelahn, International Journal of Vehicle Design, 13, 5-6, (1992).

26. Reinforced Plastics, p.36-37, Nov. (1981).

27. W.A. Whitaker, 111, 48th SPl-Cl, 13-C, (1993).

28. Reinforced Plastics, p.9, Feb, (1990).

29. I. Sigalas, M. Kumosa and D. Hull, Composite Science and Technology, 40, (1991).

30. M.J. Czaplicki et al, Composite Science and Technology, 40, (1991).

31. J.M. Gere and S.P. Timoshenko, Mechanics of Materials (Second SI Edition), Van Nostrand-Reinhold (UK) Co. Ltd, 1987.

32. Yuqiu Yang, Master thesis, Advanced Fibro-Science of Kyoto Institute of Technology, 2006. 
